\title{
Integrability and unitarity
}

\author{
Andreas Brandhuber, ${ }^{a}$ Brenda Penante, ${ }^{a}$ Gabriele Travaglini ${ }^{a, b}$ and Donovan Young ${ }^{a}$ \\ ${ }^{a}$ Centre for Research in String Theory, School of Physics and Astronomy, \\ Queen Mary University of London, \\ Mile End Road, London E1 4NS, United Kingdom \\ ${ }^{b}$ Institut für Physik und IRIS Adlershof, Humboldt-Universität zu Berlin, \\ Zum Großen Windkanal 6, 12489 Berlin, Germany \\ E-mail: a.brandhuber@qmul.ac.uk, b.penante@qmul.ac.uk, \\ g.travaglini@qmul.ac.uk, d.young@qmul.ac.uk
}

ABSTRACT: We show how generalised unitarity can be used to determine the one-loop dilatation operator in $\mathcal{N}=4$ super Yang-Mills. Our analysis focuses on two sectors, namely the bosonic $\mathrm{SO}(6)$ sector and the $\mathrm{SU}(2 \mid 3)$ sector. The calculation is performed on shell, with no off-shell information introduced at any stage. In this way, we establish a direct connection between scattering amplitudes and the dilatation operator of the $\mathcal{N}=4$ theory.

KEYwORDS: Supersymmetric gauge theory, Scattering Amplitudes, Integrable Field Theories

ArXiv EPRINT: 1502.06627v2 


\section{Contents}

1 Introduction 1

2 The dilatation operator in the $\mathrm{SO}(6)$ sector 3

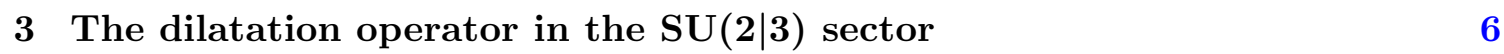

$\begin{array}{lll}4 & \text { Conclusions } & 10\end{array}$

\section{Introduction}

In this paper we continue the study initiated in [1], whose ultimate goal is to relate scattering amplitudes in $\mathcal{N}=4$ super Yang-Mills (SYM) theory, and their properties, to the dilatation operator in the same theory. In a sense, we retrace the history of the developments in the calculation of loop amplitudes triggered by Witten's twistor string theory [2]: in [1] we employed MHV diagrams [3] at loop level [4] in order to compute the dilatation operator at one loop; here, we proceed to apply a powerful variant of unitarity $[5,6]$ known as generalised unitarity $[7,8]$ which, as we shall see, allows for an even more efficient calculation of the dilatation operator. As generalised unitarity turned out be more practical than loop MHV diagrams, we will see how our use of generalised unitarity will further simplify the already remarkably simple calculation of the dilatation operator performed with MHV rules.

The use of unitarity in deriving the dilatation operator is welcome also from a conceptual point of view, since the only ingredients of the calculation are on-shell amplitudes - with no off-shell information being introduced. This supports the hope that using this approach one may be able to connect directly the amplitudes and their hidden structures and symmetries to the integrability of the dilatation operator in $\mathcal{N}=4 \mathrm{SYM}$.

At one loop, the no-triangle property [5] of the one-loop S-matrix of $\mathcal{N}=4$ SYM implies that maximal cuts employed in [8] are enough to completely determine all amplitudes of the theory. Similarly, we identify certain quadruple cuts which are sufficient to determine the dilatation operator at one loop. The reason why this is correct lies in the simplicity of the object under consideration, namely a two-point function, or a single-scale object in momentum space. At one loop there are precisely four fields to be connected, which explains why quadruple cuts are enough.

In more detail, we will focus on the dilatation operator in the $\mathrm{SO}(6)$ and $\mathrm{SU}(2 \mid 3)$ sectors of $\mathcal{N}=4 \mathrm{SYM}$, which we will derive by computing the two-point functions $\left\langle\mathcal{O}\left(x_{1}\right) \overline{\mathcal{O}}\left(x_{2}\right)\right\rangle$ of the appropriate composite operators. The $\mathrm{SO}(6)$ sector was studied in [9], where the connection to integrable spin chains was first made, and is closed (only) at one loop. The $\mathrm{SU}(2 \mid 3)$ sector is closed to all loops and was considered first in [10]. At one loop and 
in the planar limit, only contractions of pairs of adjacent fields survive. In all cases we consider, the result of our calculation turns out to be proportional to the quadruple cut of the same integral appearing in the original one-loop calculation performed by Minahan and Zarembo. This integral is given by

$$
I\left(x_{12}\right)=\int d^{D} z \Delta^{2}\left(x_{1}-z\right) \Delta^{2}\left(x_{2}-z\right),
$$

where $x_{12}:=x_{1}-x_{2}$ and

$$
\Delta(x):=-\frac{\pi^{2-\frac{D}{2}}}{4 \pi^{2}} \Gamma\left(\frac{D}{2}-1\right) \frac{1}{\left(-x^{2}+i \varepsilon\right)^{\frac{D}{2}-1}},
$$

is the scalar propagator in $D$ dimensions. In momentum space, it appears as the Fourier transform of the simplest single-scale integral, namely a double bubble,

$$
\begin{aligned}
I\left(x_{12}\right) & =\int \prod_{i=1}^{4} \frac{d^{D} L_{i}}{(2 \pi)^{D}} \frac{e^{i\left(L_{1}+L_{2}\right) \cdot x_{12}}}{L_{1}^{2} L_{2}^{2} L_{3}^{2} L_{4}^{2}}(2 \pi)^{D} \delta^{(D)}\left(\sum_{i=1}^{4} L_{i}\right) \\
& =\int \frac{d^{D} L}{(2 \pi)^{D}} e^{i L \cdot x_{12}} \int \frac{d^{D} L_{1}}{(2 \pi)^{D}} \frac{d^{D} L_{3}}{(2 \pi)^{D}} \frac{1}{L_{1}^{2}\left(L-L_{1}\right)^{2} L_{3}^{2}\left(L+L_{3}\right)^{2}},
\end{aligned}
$$

where $L:=L_{1}+L_{2}$. By using the quadruple cut where the propagators with momenta $L_{1}, L_{2}:=L-L_{1}, L_{3}$ and $L_{4}:=L+L_{3}$ are put on shell, we will be able to identify the coefficient of this double bubble in all relevant cases - without ever performing an integral. The cut double bubble can then be lifted to a full integral, and by picking its ultraviolet (UV) divergence $\left.I\left(x_{12}\right)\right|_{\mathrm{UV}}$,

$$
\left.I\left(x_{12}\right)\right|_{\mathrm{UV}}=\frac{1}{\epsilon} \cdot \frac{1}{8 \pi^{2}} \cdot \frac{1}{\left(4 \pi^{2} x_{12}^{2}\right)^{2}},
$$

we can immediately write down the dilatation operator. Let us also mention that other applications of unitarity to the calculation of $n$-point correlators and correlation functions of Wilson lines have appeared in [11-13]. It is worth stressing the two key reasons why generalised unitarity is particularly powerful for the case considered here, namely that of the two-point function. First, as we have already mentioned, quadruple cuts are precisely the right set of cuts to identify the relevant loop integral; furthermore, the relevant integrals are guaranteed to have a single scale. The cut integral can then be lifted to a full loop integral without introducing spurious discontinuities, in complete analogy to the case of splitting amplitudes studied in [14]. Finally, we also note that our approach to the computation of the dilatation operator differs from that of $[15,16]$ in that no infrared divergences appear at any stage in our calculation.

The rest of the paper is organised as follows. In the next section we use generalised unitarity to obtain the dilatation operator in the $\mathrm{SO}(6)$ sector. In section 3 we move on to the $\mathrm{SU}(2 \mid 3)$ sector. This case is particularly interesting as it involves fermions as well as scalars. There are several contributions to consider and the structure of the dilatation operator is more elaborate than in the pure scalar sector, hence our tests are more stringent. Finally in section 4 we compare recent on-shell and twistorial approaches for the calculation of the dilatation operator, and also make a few suggestions for future work. 


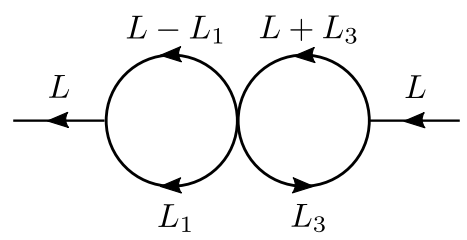

Figure 1. The double-bubble integral relevant for the computation of $I\left(x_{12}\right)$.

\section{The dilatation operator in the $\mathrm{SO}(6)$ sector}

In this section we will compute the dilatation operator of $\mathcal{N}=4 \mathrm{SYM}$ in the $\mathrm{SO}(6)$ sector using generalised unitarity. This calculation was recently performed in [17] and [1] using MHV diagrams in twistor space and momentum space, respectively. Here we depart from these off-shell approaches in favour of a fully on-shell calculation.

Operators in the $\mathrm{SO}(6)$ sector have the form

$$
\mathcal{O}_{A_{1} B_{1}, A_{2} B_{2}, \ldots, A_{L} B_{L}}(x):=\operatorname{Tr}\left(\phi_{A_{1} B_{1}}(x) \cdots \phi_{A_{L} B_{L}}(x)\right) .
$$

At one loop and in the planar limit, it is sufficient to consider contractions of pairs of adjacent fields (in colour space). The relevant part of each operator is then

$$
\begin{aligned}
& \mathcal{O}\left(x_{1}\right)=\cdots \phi_{A B}^{a}\left(x_{1}\right) \phi_{C D}^{b}\left(x_{1}\right) \cdots\left(T^{a} T^{b}\right)_{j}^{i}, \\
& \overline{\mathcal{O}}\left(x_{2}\right)=\cdots \phi_{A^{\prime} B^{\prime}}^{c}\left(x_{2}\right) \phi_{C^{\prime} D^{\prime}}^{d}\left(x_{2}\right) \cdots\left(T^{c} T^{d}\right)_{m}^{l} .
\end{aligned}
$$

The calculation is then effectively equivalent to that of the following two-point function

$$
\left\langle\left(\phi_{A B}^{a} \phi_{C D}^{b}\right)\left(x_{1}\right)\left(\phi_{A^{\prime} B^{\prime}}^{c} \phi_{C^{\prime} D^{\prime}}^{d}\right)\left(x_{2}\right)\right\rangle,
$$

whose expected structure is

$$
\begin{aligned}
& \left\langle\left(\phi_{A B} \phi_{C D}\right)\left(x_{1}\right)\left(\phi_{A^{\prime} B^{\prime}} \phi_{C^{\prime} D^{\prime}}\right)\left(x_{2}\right)\right\rangle \\
& =\mathcal{A} \epsilon_{A B C D} \epsilon_{A^{\prime} B^{\prime} C^{\prime} D^{\prime}}+\mathcal{B} \epsilon_{A B A^{\prime} B^{\prime}} \epsilon_{C D C^{\prime} D^{\prime}}+\mathcal{C} \epsilon_{A B C^{\prime} D^{\prime} \epsilon_{A^{\prime} B^{\prime} C D} .}
\end{aligned}
$$

These three terms are usually referred to as trace, permutation and identity. The dilatation operator can then be read off from the UV divergences of (2.4), hence we only need to compute the UV-divergent parts $\mathcal{A}_{\mathrm{UV}}, \mathcal{B}_{\mathrm{UV}}, \mathcal{C}_{\mathrm{UV}}$ of the coefficients $\mathcal{A}, \mathcal{B}$ and $\mathcal{C}$. These are expected to be equal to [9]

$$
\mathcal{A}_{\mathrm{UV}}=\frac{1}{2}, \quad \mathcal{B}_{\mathrm{UV}}=-1, \quad \mathcal{C}_{\mathrm{UV}}=1 .
$$

As in [1], we choose the following SU(4) assignments in (2.2) as representatives of these three flavour structures:

\begin{tabular}{|c|cc|}
\hline & $A B C D$ & $A^{\prime} B^{\prime} C^{\prime} D^{\prime}$ \\
\hline $\operatorname{Tr}$ & 1234 & 2413 \\
$\mathbb{P}$ & 1213 & 3424 \\
$\mathbb{1}$ & 1213 & 2434 \\
\hline
\end{tabular}




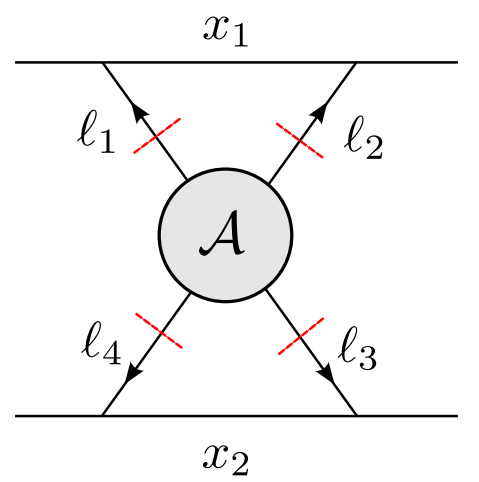

Figure 2. The single cut diagram contributing to the dilatation operator at one loop.

For each case there is a single cut diagram to consider. The integrand is constructed with four cut scalar propagators with momenta $L_{i}, i=1, \ldots, 4$, and one on-shell amplitude, as shown in figure 2. The operators are connected to the amplitude via appropriate form factors, which in the scalar case are simply

$$
\begin{aligned}
F_{\phi^{a} \tilde{\phi}^{b}}\left(\ell_{1}^{\phi^{a^{\prime}}}, \ell_{2}^{\tilde{\phi}^{b^{\prime}}} ; L\right) & :=\int d^{4} x e^{i L \cdot x}\left\langle 0\left|\left(\phi^{a} \tilde{\phi}^{b}\right)(x)\right| \phi^{a^{\prime}}\left(\ell_{1}\right), \tilde{\phi}^{b^{\prime}}\left(\ell_{2}\right)\right\rangle \\
& =(2 \pi)^{4} \delta^{(4)}\left(L-\ell_{1}-\ell_{2}\right) \delta^{a a^{\prime}} \delta^{b b^{\prime}},
\end{aligned}
$$

where we have used $\phi$ and $\tilde{\phi}$ to denote two scalar fields having distinct $R$-symmetry indices as is sufficient for our purposes, see (2.6). Note that the $\ell_{i}$ represent the on-shell (cut) versions of the loop momenta $L_{i}$.

The relevant amplitudes for the three flavour assignments considered in (2.6) are: ${ }^{1}$

$$
\begin{array}{ll}
\text { Tr : } & A\left(1^{\phi_{12}}, 4^{\phi_{13}}, 3^{\phi_{24}}, 2^{\phi_{34}}\right)=\frac{\langle 13\rangle\langle 24\rangle}{\langle 12\rangle\langle 34\rangle}, \\
\mathbb{P}: & A\left(1^{\phi_{12}}, 4^{\phi_{24}}, 3^{\phi_{34}}, 2^{\phi_{13}}\right)=-1, \\
\mathbb{1}: & A\left(1^{\phi_{12}}, 4^{\phi_{34}}, 3^{\phi_{24}}, 2^{\phi_{13}}\right)=\frac{\langle 13\rangle\langle 24\rangle}{\langle 23\rangle\langle 14\rangle} .
\end{array}
$$

Three observations are in order here. First, we note that the same integrands as in the approach of [1] have appeared, with the important difference that, in that paper, the spinors associated with the on-shell momenta are given by the appropriate off-shell continuation for MHV diagrams. Here the spinors for the cut loop momenta do not need any off-shell continuation. Furthermore, for the case of the $\mathbb{P}$ integrand there is obviously no difference between the two approaches, and the resulting integral is given by a double bubble where all the four propagators are cut. In the other two cases, this integral is dressed by the appropriate amplitude. Finally, we note that the colour factor associated with all diagrams is obtained from the contraction

$$
\cdots\left(T^{b} T^{a}\right)_{j}^{i} \cdots \operatorname{Tr}\left(T^{a} T^{b} T^{c} T^{d}\right) \cdots\left(T^{d} T^{c}\right)_{m}^{l} \cdots=\cdots N^{2} \delta_{m}^{i} \delta_{j}^{l} \cdots,
$$

\footnotetext{
${ }^{1}$ In the following expressions we omit a factor of $g_{\mathrm{YM}}^{2}$, which will be reintroduced at the end of the calculation.
} 
where the trace arises from the amplitude and the factors $\cdots\left(T^{b} T^{a}\right)_{j}^{i} \cdots$ and $\cdots\left(T^{d} T^{c}\right)_{m}^{l} \cdots$ from the operators (and we indicate only generators corresponding to the fields being contracted). We now proceed to construct the relevant integrands.

The trace integrand. In this case the relevant amplitude (which multiplies four cut propagators) can be rewritten as $^{2}$

$$
\frac{\langle 13\rangle\langle 24\rangle}{\langle 12\rangle\langle 34\rangle}=\frac{\operatorname{Tr}_{+}\left(\ell_{1} \ell_{3} \ell_{4} \ell_{2}\right)}{\left(\ell_{1}+\ell_{2}\right)^{2}\left(\ell_{3}+\ell_{4}\right)^{2}}=-\frac{2\left(\ell_{1} \cdot \ell_{3}\right)}{L^{2}},
$$

where we have used $\ell_{1}+\ell_{2}=-\left(\ell_{3}+\ell_{4}\right):=L$. Having rewritten the amplitude in terms of products of momenta, we lift the four cut momenta off shell. The resulting integral has the structure of a product of two linear bubbles,

$$
-\frac{2}{L^{2}} \int \frac{d^{D} L_{1}}{(2 \pi)^{D}} \frac{L_{1}^{\mu}}{L_{1}^{2}\left(L-L_{1}\right)^{2}} \int \frac{d^{D} L_{3}}{(2 \pi)^{D}} \frac{L_{3 \mu}}{L_{3}^{2}\left(L+L_{3}\right)^{2}} .
$$

Using the fact that

$$
\int \frac{d^{D} K}{(2 \pi)^{D}} \frac{K^{\mu}}{K^{2}(K \pm L)^{2}}=\mp \frac{L^{\mu}}{2} \operatorname{Bub}\left(L^{2}\right)
$$

where

$$
\operatorname{Bub}\left(L^{2}\right):=\int \frac{d^{D} K}{(2 \pi)^{D}} \frac{1}{K^{2}(K+L)^{2}},
$$

we find that (2.13) is equal to $1 / 2$ times a double bubble. Using (1.4) we finally arrive at $\mathcal{A}_{\mathrm{UV}}=1 / 2$. Note that in the definitions of $\mathcal{A}_{\mathrm{UV}}, \mathcal{B}_{\mathrm{UV}}$, and $\mathcal{C}_{\mathrm{UV}}$, a factor of $\lambda /\left(8 \pi^{2}\right) \times$ $\left(1 /\left(4 \pi^{2} x_{12}^{2}\right)\right)^{2} \times(1 / \epsilon)$ will always be understood, with $\lambda:=g_{\mathrm{YM}}^{2} N$.

The $\mathbb{P}$ integrand. No calculation is needed in this case, and the result is simply given by minus a cut double-bubble integral. Lifting the cut integral to a full loop integral we get $\mathcal{B}_{\mathrm{UV}}=-1$.

The $\mathbb{1}$ integrand. The relevant amplitude in this case is

$$
\frac{\langle 13\rangle\langle 24\rangle}{\langle 23\rangle\langle 14\rangle}=1+\frac{\langle 12\rangle\langle 34\rangle}{\langle 23\rangle\langle 14\rangle}
$$

Thus the first term in (2.16) gives the cut double-bubble integral, whereas we can use on-shell identities to rewrite the second term as

$$
\frac{\langle 12\rangle\langle 34\rangle}{\langle 23\rangle\langle 14\rangle}=\frac{\langle 12\rangle\langle 34\rangle[34]}{\langle 23\rangle\langle 14\rangle[34]}=-\frac{L^{2}}{2\left(\ell_{1} \cdot \ell_{4}\right)} .
$$

Lifting the cut propagators of the second integral to full propagators, it is immediate to see that this term produces the integral represented in figure 3. This integral is finite in four dimensions and thus does not contribute to $\mathcal{C}_{\mathrm{UV}}$. We then conclude that $\mathcal{C}_{\mathrm{UV}}=1$. For later convenience, we explicitly write down the form of the UV-divergent part of the

\footnotetext{
${ }^{2}$ We define $\operatorname{Tr}_{+}(a b c d):=\langle a b\rangle[b c]\langle c d\rangle[d a]$.
} 


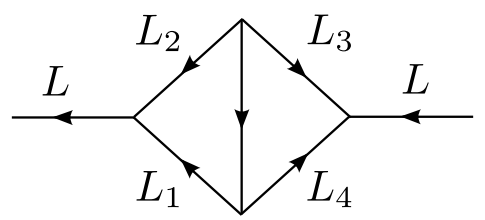

Figure 3. The finite integral corresponding to the term in (2.17). This integral is irrelevant for the calculation of the dilatation operator.

correlator (2.4),

$$
\begin{aligned}
& \left.\left\langle\left(\phi_{A B} \phi_{C D}\right)_{j}^{i}\left(x_{1}\right)\left(\phi_{A^{\prime} B^{\prime}} \phi_{C^{\prime} D^{\prime}}\right)_{m}^{l}\left(x_{2}\right)\right\rangle\right|_{\mathrm{UV}} \\
& \quad=\frac{1}{\epsilon} \cdot \frac{\lambda}{8 \pi^{2}}\left(\Delta^{2}\left(x_{12}\right) \delta_{m}^{i} \delta_{j}^{l}\right)\left(\frac{1}{2} \epsilon_{A B C D} \epsilon_{A^{\prime} B^{\prime} C^{\prime} D^{\prime}}-\epsilon_{A B A^{\prime} B^{\prime}} \epsilon_{C D C^{\prime} D^{\prime}}+\epsilon_{A B C^{\prime} D^{\prime}} \epsilon_{A^{\prime} B^{\prime} C D}\right) .
\end{aligned}
$$

In terms of a spin-chain Hamiltonian, this can be represented as [9]

$$
H_{2}=\frac{\lambda}{8 \pi^{2}}\left(\frac{1}{2} \operatorname{Tr}+\mathbb{1}-\mathbb{P}\right)
$$

\section{The dilatation operator in the $\mathrm{SU}(2 \mid 3)$ sector}

In this section we consider the closed $\mathrm{SU}(2 \mid 3)$ sector. This is particularly interesting, as it involves also fermions. Indeed, operators in this sector are formed with letters taken from the set $\left\{\psi_{1 \alpha}, \phi_{1 A}\right\}$, with $\alpha=1,2$ and $A=2,3,4$. We thus have one fermion and three scalar fields. The dilatation operator in this sector was derived in [10]. Its expression is given by

$$
H_{2}=\frac{\lambda}{8 \pi^{2}}\left[\left\{\begin{array}{l}
A B \\
A B
\end{array}\right\}-\left\{\begin{array}{l}
A B \\
B A
\end{array}\right\}+\left\{\begin{array}{l}
A \beta \\
A \beta
\end{array}\right\}+\left\{\begin{array}{l}
\alpha B \\
\alpha B
\end{array}\right\}-\left(\left\{\begin{array}{l}
A \beta \\
\beta A
\end{array}\right\}+\left\{\begin{array}{l}
\alpha B \\
B \alpha
\end{array}\right\}\right)+\left\{\begin{array}{l}
\alpha \beta \\
\alpha \beta
\end{array}\right\}+\left\{\begin{array}{c}
\alpha \beta \\
\beta \alpha
\end{array}\right\}\right]
$$

where in this notation the three scalar fields are labelled by $A, B=2,3,4$. In the following we are going to rederive (3.1) using an application of generalised unitarity.

As for the $\mathrm{SO}(6)$ case, in the planar limit only contractions between nearest-neighbour fields in $\mathcal{O}\left(x_{1}\right)$ and $\overline{\mathcal{O}}\left(x_{2}\right)$ have to be considered. The first two terms on the right-hand side of (3.1) denote the scalar identity $\mathbb{1}$ and permutation $\mathbb{P}$ structures already familiar from the $\mathrm{SO}(6)$ case (the trace structure is absent given the restricted choice of scalar letters). The novelty is that now we have to consider two additional types of contractions: scalarfermion $\rightarrow$ scalar-fermion, and two-fermion $\rightarrow$ two-fermion, as indicated in the remaining terms in (3.1).

Scalar-fermion $\rightarrow$ scalar-fermion. In this case we are interested in a fermion field $\psi_{1 \alpha}$ and one of the scalars $\phi_{12}, \phi_{13}$, or $\phi_{14}$. Without loss of generality we will consider $\phi_{12}$. There are two cases to consider,

$$
\mathbf{U}: \quad\left\langle\left(\phi_{12}^{a} \psi_{1 \alpha}^{b}\right)\left(x_{1}\right)\left(\psi_{234 \dot{\alpha}}^{c} \phi_{34}^{d}\right)\left(x_{2}\right)\right\rangle,
$$


and

$$
\mathbf{S}: \quad\left\langle\left(\phi_{12}^{a} \psi_{1 \alpha}^{b}\right)\left(x_{1}\right)\left(\phi_{34}^{c} \psi_{234 \dot{\alpha}}^{d}\right)\left(x_{2}\right)\right\rangle,
$$

where the letters $\mathbf{U}$ and $\mathbf{S}$ indicate whether the contractions between the two fields are unswapped or swapped. The relevant form factor is

$$
\begin{aligned}
F_{\phi_{12}^{a} \psi_{1 \alpha}^{b}}\left(\ell_{1}^{\phi_{12}^{a^{\prime}}}, \ell_{2}^{\psi_{1 \alpha}^{b^{\prime}}} ; L\right) & :=\int d^{4} x e^{i L \cdot x}\left\langle 0\left|\left(\phi_{12}^{a} \psi_{1 \alpha}^{b}\right)(x)\right| \phi_{12}^{a^{\prime}}\left(\ell_{1}\right), \psi_{1}^{b^{\prime}}\left(\ell_{2}\right)\right\rangle \\
& =(2 \pi)^{4} \delta^{(4)}\left(L-\ell_{1}-\ell_{2}\right) \lambda_{\alpha}^{2} \delta^{a a^{\prime}} \delta^{b b^{\prime}},
\end{aligned}
$$

and similarly for $\overline{\mathcal{O}}\left(x_{2}\right)$.

We begin by considering the $\mathbf{U}$ case. By contracting the two form factors with the four planar permutations of the full amplitude, we obtain ${ }^{3}$

$$
\begin{aligned}
& \lambda_{\alpha}^{2} \widetilde{\lambda}_{\dot{\alpha}}^{3} \delta^{a a^{\prime}} \delta^{b b^{\prime}} \delta^{c c^{\prime}} \delta^{d d^{\prime}} \\
& \quad \times\left[A\left(1^{\phi_{12}}, 2^{\psi_{1}}, 3^{\psi_{234}}, 4^{\phi_{34}}\right) \operatorname{Tr}\left(T^{a^{\prime}} T^{b^{\prime}} T^{c^{\prime}} T^{d^{\prime}}\right)+A\left(1^{\phi_{12}}, 2^{\psi_{1}}, 4^{\phi_{34}}, 3^{\psi_{234}}\right) \operatorname{Tr}\left(T^{a^{\prime}} T^{b^{\prime}} T^{d^{\prime}} T^{c^{\prime}}\right)\right. \\
& \left.\quad-A\left(1^{\phi_{12}}, 3^{\psi_{234}}, 4^{\phi_{34}}, 2^{\psi_{1}}\right) \operatorname{Tr}\left(T^{a^{\prime}} T^{c^{\prime}} T^{d^{\prime}} T^{b^{\prime}}\right)-A\left(1^{\phi_{12}}, 4^{\phi_{34}}, 3^{\psi_{234}}, 2^{\psi_{1}}\right) \operatorname{Tr}\left(T^{a^{\prime}} T^{d^{\prime}} T^{c^{\prime}} T^{b^{\prime}}\right)\right] .
\end{aligned}
$$

At large $N$ there is only one leading contribution, corresponding to the term with the amplitude

$$
A\left(1^{\phi_{12}}, 4^{\phi_{34}}, 3^{\psi_{234}}, 2^{\psi_{1}}\right)=\frac{\langle 13\rangle\langle 34\rangle}{\langle 14\rangle\langle 23\rangle} .
$$

It is given by

$$
-A\left(1^{\phi_{12}}, 4^{\phi_{34}}, 3^{\psi_{234}}, 2^{\psi_{1}}\right) \lambda_{\alpha}^{2} \widetilde{\lambda}_{\dot{\alpha}}^{3}=-\frac{\left(\ell_{2} \bar{\ell}_{1} \ell_{3}\right)_{\alpha \dot{\alpha}}}{2\left(\ell_{1} \cdot \ell_{4}\right)}:=N_{\alpha \dot{\alpha}}
$$

The cut integral to consider is thus

$$
I_{\alpha \dot{\alpha}}:=\int d^{4} \ell_{1} d^{4} \ell_{3} \delta^{(+)}\left(\ell_{1}^{2}\right) \delta^{(+)}\left(\ell_{3}^{2}\right) \delta^{(+)}\left(\left(L-\ell_{1}\right)^{2}\right) \delta^{(+)}\left(\left(L+\ell_{3}\right)^{2}\right) \cdot N_{\alpha \dot{\alpha}},
$$

where by Lorentz invariance $I_{\alpha \dot{\alpha}}$ must have the form

$$
I_{\alpha \dot{\alpha}}=A L_{\alpha \dot{\alpha}} .
$$

A simple PV reduction shows that $A=1 / 2$.

For the $\mathbf{S}$ case, we get the single leading contribution to be

$$
-A\left(1^{\phi_{12}}, 4^{\psi_{234}}, 3^{\phi_{34}}, 2^{\psi_{1}}\right) \lambda_{\alpha}^{2} \widetilde{\lambda}_{\dot{\alpha}}^{4}=-\frac{\left(\ell_{2} \bar{\ell}_{1} \ell_{4}\right)_{\alpha \dot{\alpha}}}{2\left(\ell_{2} \cdot \ell_{3}\right)}:=\tilde{N}_{\alpha \dot{\alpha}} .
$$

The relevant integral is now

$$
\begin{aligned}
\tilde{I}_{\alpha \dot{\alpha}} & :=\int d^{4} \ell_{1} d^{4} \ell_{3} \delta^{(+)}\left(\ell_{1}^{2}\right) \delta^{(+)}\left(\ell_{3}^{2}\right) \delta^{(+)}\left(\left(L-\ell_{1}\right)^{2}\right) \delta^{(+)}\left(\left(L+\ell_{3}\right)^{2}\right) \cdot \tilde{N}_{\alpha \dot{\alpha}} \\
& =\tilde{A} L_{\alpha \dot{\alpha}},
\end{aligned}
$$

\footnotetext{
${ }^{3}$ Two out of the six possible contractions obviously do not contribute at large $N$.
} 
where a PV reduction shows that $\tilde{A}=-1 / 2$. Note that in arriving at this result we have discarded finite integrals, which do not contribute to the anomalous dimensions (more precisely, in all calculations the only other finite integral appearing is the kite, depicted in figure 3).

Summarising, the scalar-fermion $\rightarrow$ scalar-fermion case gives $\pm 1 / 2 L_{\alpha \dot{\alpha}}$ times a doublebubble integral, for the $\mathbf{U} / \mathbf{S}$ case, respectively. This has to be compared to the tree-level expression

$$
I_{\alpha \dot{\alpha}}^{\text {tree }}:=\int \frac{d^{D} L_{1}}{(2 \pi)^{D}} \frac{L_{1 \alpha \dot{\alpha}}}{L_{1}^{2}\left(L-L_{1}\right)^{2}}=\frac{1}{2} L_{\alpha \dot{\alpha}}
$$

Thus for the two-scalar two-fermion case we get:

$$
\mathbb{1}: 1, \quad \mathbb{P}:-1,
$$

and the corresponding contribution to the spin-chain Hamiltonian is ${ }^{4}$

$$
\frac{\lambda}{8 \pi^{2}}\left(\left\{\begin{array}{l}
A \beta \\
A \beta
\end{array}\right\}-\left\{\begin{array}{c}
A \beta \\
\beta A
\end{array}\right\}\right)
$$

in agreement with the corresponding terms in (3.1).

Two-fermion $\rightarrow$ two-fermion. In this case we consider the four-point correlator

$$
\left\langle\left(\psi_{1 \alpha}^{a} \psi_{1 \beta}^{b}\right)\left(x_{1}\right)\left(\psi_{234 \dot{\alpha}}^{c} \psi_{234 \dot{\beta}}^{d}\right)\left(x_{2}\right)\right\rangle \text {. }
$$

The form factors of $\mathcal{O}\left(x_{1}\right)$ are given by

$$
\begin{aligned}
F_{\psi_{1 \alpha}^{a} \psi_{1 \beta}^{b}}\left(\ell_{1 \alpha}^{\psi_{1 \alpha}^{a^{\prime}}}, \ell_{2}^{\psi_{1 \beta}^{b^{\prime}}} ; L\right) & :=\int d^{4} x e^{i L \cdot x}\left\langle 0\left|\left(\psi_{1 \alpha}^{a} \psi_{1 \beta}^{b}\right)\left(x_{1}\right)\right| \psi_{1}^{a^{\prime}}\left(\ell_{1}\right), \psi_{1}^{b^{\prime}}\left(\ell_{2}\right)\right\rangle \\
& =(2 \pi)^{4} \delta^{(4)}\left(L-\ell_{1}-\ell_{2}\right) \cdot \frac{1}{2}\left(\lambda_{\alpha}^{1} \lambda_{\beta}^{2} \delta^{a a^{\prime}} \delta^{b b^{\prime}}-\lambda_{\beta}^{1} \lambda_{\alpha}^{2} \delta^{a b^{\prime}} \delta^{b a^{\prime}}\right),
\end{aligned}
$$

and similarly for the form factor of $\overline{\mathcal{O}}\left(x_{2}\right)$. Note the factor of $1 / 2$ appearing because of the presence of two identical particles in the state. Contracting the two form factors with the four planar permutations of the full amplitude, we get

$$
\begin{aligned}
- & \frac{1}{4}\left(\lambda_{\alpha}^{1} \lambda_{\beta}^{2} \delta^{a a^{\prime}} \delta^{b b^{\prime}}-\lambda_{\beta}^{1} \lambda_{\alpha}^{2} \delta^{a b^{\prime}} \delta^{b a^{\prime}}\right)\left(\widetilde{\lambda}_{\dot{\alpha}}^{3} \widetilde{\lambda}_{\dot{\beta}}^{4} \delta^{c c^{\prime}} \delta^{d d^{\prime}}-\widetilde{\lambda}_{\dot{\beta}}^{3} \widetilde{\lambda}_{\dot{\alpha}}^{4} \delta^{c d^{\prime}} \delta^{d c^{\prime}}\right) \\
& \times\left[A\left(1^{\psi_{1}}, 2^{\psi_{1}}, 3^{\psi_{234}}, 4^{\psi_{234}}\right) \operatorname{Tr}\left(T^{a^{\prime}} T^{b^{\prime}} T^{c^{\prime}} T^{d^{\prime}}\right)-A\left(1^{\psi_{1}}, 2^{\psi_{1}}, 4^{\psi_{234}}, 3^{\psi_{234}}\right) \operatorname{Tr}\left(T^{a^{\prime}} T^{b^{\prime}} T^{d^{\prime}} T^{c^{\prime}}\right)\right. \\
& \left.+A\left(1^{\psi_{1}}, 3^{\psi_{234}}, 4^{\psi_{234}}, 2^{\psi_{1}}\right) \operatorname{Tr}\left(T^{a^{\prime}} T^{c^{\prime}} T^{d^{\prime}} T^{b^{\prime}}\right)-A\left(1^{\psi_{1}}, 4^{\psi_{234}}, 3^{\psi_{234}}, 2^{\psi_{1}}\right) \operatorname{Tr}\left(T^{a^{\prime}} T^{d^{\prime}} T^{c^{\prime}} T^{b^{\prime}}\right)\right] .
\end{aligned}
$$

In the large- $N$ limit we only need to keep the following terms out of those in (3.17):

$$
\begin{aligned}
-\frac{1}{4}[ & A\left(1^{\psi_{1}}, 2^{\psi_{1}}, 3^{\psi_{234}}, 4^{\psi_{234}}\right) \lambda_{\beta}^{1} \lambda_{\alpha}^{2} \widetilde{\lambda}_{\dot{\beta}}^{3} \widetilde{\lambda}_{\dot{\alpha}}^{4}+A\left(1^{\psi_{1}}, 2^{\psi_{1}}, 4^{\psi_{234}}, 3^{\psi_{234}}\right) \lambda_{\beta}^{1} \lambda_{\alpha}^{2} \widetilde{\lambda}_{\dot{\alpha}}^{3} \widetilde{\lambda}_{\dot{\beta}}^{4} \\
& \left.-A\left(1^{\psi_{1}}, 3^{\psi_{234}}, 4^{\psi_{234}}, 2^{\psi_{1}}\right) \lambda_{\alpha}^{1} \lambda_{\beta}^{2} \widetilde{\lambda}_{\dot{\beta}}^{3} \widetilde{\lambda}_{\dot{\alpha}}^{4}-A\left(1^{\psi_{1}}, 4^{\psi_{234}}, 3^{\psi_{234}}, 2^{\psi_{1}}\right) \lambda_{\alpha}^{1} \lambda_{\beta}^{2} \widetilde{\lambda}_{\dot{\alpha}}^{3} \widetilde{\lambda}_{\dot{\beta}}^{4}\right],
\end{aligned}
$$

\footnotetext{
${ }^{4}$ Here we also reinstate powers of $g_{\mathrm{YM}}^{2}$ from the tree-level amplitudes, of $N$, arising from colour contractions, and a factor of $1 /\left(8 \pi^{2}\right)$ arising from the UV singularity (1.4) of the double-bubble integral (1.3).
} 
where the relevant four-fermion amplitudes are

$$
\begin{aligned}
& A\left(1^{\psi_{1}}, 2^{\psi_{1}}, 3^{\psi_{234}}, 4^{\psi_{234}}\right)=-\frac{\langle 34\rangle^{2}}{\langle 23\rangle\langle 41\rangle}, \\
& A\left(1^{\psi_{1}}, 2^{\psi_{1}}, 4^{\psi_{234}}, 3^{\psi_{234}}\right)=-\frac{\langle 34\rangle^{2}}{\langle 24\rangle\langle 31\rangle}, \\
& A\left(1^{\psi_{1}}, 3^{\psi_{234}}, 4^{\psi_{234}}, 2^{\psi_{1}}\right)=\frac{\langle 34\rangle^{2}}{\langle 13\rangle\langle 42\rangle}, \\
& A\left(1^{\psi_{1}}, 4^{\psi_{234}}, 3^{\psi_{234}}, 2^{\psi_{1}}\right)=\frac{\langle 34\rangle^{2}}{\langle 14\rangle\langle 32\rangle} .
\end{aligned}
$$

Using (3.19), we can rewrite (3.18) as

$$
\frac{1}{4}\left[\frac{\left(\ell_{2} \overline{\ell_{1}}\right)_{\alpha \beta}\left(\overline{\ell_{4}} \ell_{3}\right)_{\dot{\alpha} \dot{\beta}}+\left(\ell_{1} \overline{\ell_{2}}\right)_{\alpha \beta}\left(\overline{\ell_{3}} \ell_{4}\right)_{\dot{\alpha} \dot{\beta}}}{2\left(\ell_{2} \cdot \ell_{3}\right)}+\ell_{1} \leftrightarrow \ell_{2}\right] .
$$

The term with $\ell_{1} \leftrightarrow \ell_{2}$ is simply a relabelling of the integration variables, and we conclude that the one-loop integrand is given by

$$
\frac{1}{2}\left[\frac{\left(\ell_{2} \bar{\ell}_{1}\right)_{\alpha \beta}\left(\bar{\ell}_{4} \ell_{3}\right)_{\dot{\alpha} \dot{\beta}}+\left(\ell_{1} \bar{\ell}_{2}\right)_{\alpha \beta}\left(\overline{\ell_{3}} \ell_{4}\right)_{\dot{\alpha} \dot{\beta}}}{2\left(\ell_{2} \cdot \ell_{3}\right)}\right] \equiv N_{\alpha \beta \dot{\alpha} \dot{\beta}} .
$$

Thus we have to consider the cut-integral

$$
I_{\alpha \beta \dot{\alpha} \dot{\beta}}:=\int d^{4} \ell_{1} d^{4} \ell_{3} \delta^{(+)}\left(\ell_{1}^{2}\right) \delta^{(+)}\left(\ell_{3}^{2}\right) \delta^{(+)}\left(\left(L-\ell_{1}\right)^{2}\right) \delta^{(+)}\left(\left(L+\ell_{3}\right)^{2}\right) \cdot N_{\alpha \beta \dot{\alpha} \dot{\beta}} .
$$

It depends on only one scale $L$, hence it has the form

$$
I_{\alpha \beta \dot{\alpha} \dot{\beta}}=A L^{2} \epsilon_{\alpha \beta} \epsilon_{\dot{\alpha} \dot{\beta}}+B\left(L_{\alpha \dot{\alpha}} L_{\beta \dot{\beta}}+L_{\alpha \dot{\beta}} L_{\beta \dot{\alpha}}\right) \text {. }
$$

Contracting (3.22) and (3.23) with $\epsilon^{\alpha \beta} \epsilon^{\dot{\alpha} \dot{\beta}}$ and $\left(\bar{L}^{\dot{\alpha} \alpha} \bar{L}^{\dot{\beta} \beta}+\bar{L}^{\dot{\beta} \alpha} \bar{L}^{\dot{\alpha} \beta}\right)$ we can solve for the coefficients $A$ and $B$, with the result

$$
A=0, \quad B=1 / 6 .
$$

At this point we lift the four cut propagators to full propagators, so that the cut double bubble becomes a full double-bubble integral. The conclusion is then that the UV divergent part of the integral representing the two-fermion $\rightarrow$ two-fermion process is a double bubble with coefficient

$$
\frac{1}{6}\left(L_{\alpha \dot{\alpha}} L_{\beta \dot{\beta}}+L_{\alpha \dot{\beta}} L_{\beta \dot{\alpha}}\right) .
$$

This result has to be compared with the planar contractions at tree level,

$$
I_{\alpha \beta \dot{\alpha} \dot{\beta}}^{\text {tree }}:=\int \frac{d^{D} L_{1}}{(2 \pi)^{D}} \frac{L_{1 \alpha \dot{\beta}}\left(L-L_{1}\right)_{\beta \dot{\alpha}}}{L_{1}^{2}\left(L-L_{1}\right)^{2}} .
$$

After a similar PV reduction of the $L_{1}$ integration in (3.26), we find that $I_{\alpha \beta \dot{\alpha} \dot{\beta}}^{\text {tree }}$ is given by a scalar (single) bubble with coefficient

$$
\frac{1}{6}\left(-L^{2} \epsilon_{\alpha \beta} \epsilon_{\dot{\alpha} \dot{\beta}}+L_{\alpha \dot{\beta}} L_{\beta \dot{\alpha}}\right),
$$


which using $L_{\alpha \dot{\alpha}} L_{\beta \dot{\beta}}-L_{\beta \dot{\alpha}} L_{\alpha \dot{\beta}}=L^{2} \epsilon_{\alpha \beta} \epsilon_{\dot{\alpha} \dot{\beta}}$ can be rewritten as

$$
\frac{1}{4}\left[-L^{2} \epsilon_{\alpha \beta} \epsilon_{\dot{\alpha} \dot{\beta}}+\frac{1}{3}\left(L_{\alpha \dot{\alpha}} L_{\beta \dot{\beta}}+L_{\beta \dot{\alpha}} L_{\alpha \dot{\beta}}\right)\right]
$$

This is the "identity" or $\left\{\begin{array}{c}\alpha \beta \\ \alpha \beta\end{array}\right\}$. The permutation is obtained by swapping $\dot{\alpha}$ and $\dot{\beta}$, or $\left\{\begin{array}{c}\alpha \beta \\ \beta \alpha\end{array}\right\}$. Thus, we can write:

$$
\begin{aligned}
& \left\{\begin{array}{c}
\alpha \beta \\
\alpha \beta
\end{array}\right\}: \frac{1}{4}\left[-L^{2} \epsilon_{\alpha \beta} \epsilon_{\dot{\alpha} \dot{\beta}}+\frac{1}{3}\left(L_{\alpha \dot{\alpha}} L_{\beta \dot{\beta}}+L_{\beta \dot{\alpha}} L_{\alpha \dot{\beta}}\right)\right], \\
& \left\{\begin{array}{c}
\alpha \beta \\
\beta \alpha
\end{array}\right\}: \frac{1}{4}\left[L^{2} \epsilon_{\alpha \beta} \epsilon_{\dot{\alpha} \dot{\beta}}+\frac{1}{3}\left(L_{\alpha \dot{\alpha}} L_{\beta \dot{\beta}}+L_{\beta \dot{\alpha}} L_{\alpha \dot{\beta}}\right)\right] .
\end{aligned}
$$

In this language, the tree-level contraction is represented as

$$
\left\{\begin{array}{c}
\alpha \beta \\
\alpha \beta
\end{array}\right\} \text {. }
$$

Hence, also reinstating powers of the 't Hooft coupling, we obtain that the term in the spin-chain Hamiltonian corresponding to the two-fermion $\rightarrow$ two-fermion process is

$$
\frac{\lambda}{8 \pi^{2}}\left(\left\{\begin{array}{c}
\alpha \beta \\
\alpha \beta
\end{array}\right\}+\left\{\begin{array}{c}
\alpha \beta \\
\beta \alpha
\end{array}\right\}\right)
$$

in agreement with the corresponding terms in (3.1). In conclusion, putting together the purely scalar result of section 2, (2.19), as well as the results (3.14) and (3.32) for the twofermion two-scalar and four-fermion cases, we have confirmed the complete expression (3.1) for the spin-chain Hamiltonian in the $\mathrm{SU}(2 \mid 3)$ sector.

\section{Conclusions}

We would like to summarise some of the key points of our paper, compare with other recent on-shell approaches and finally make a few suggestions for future research.

The calculation of the dilatation operator in $\mathcal{N}=4 \mathrm{SYM}$ has been revisited in recent months using on-shell and twistor (string) inspired approaches. In [15] the complete oneloop dilatation operator was obtained by calculating form factors for generic single-trace operators using unitarity, making interesting contact with earlier work of [18]. In particular, the integral form for the dilatation operator in [18] is mapped to a phase-space integral, which appears naturally in a unitarity-based approach. The calculation of two-loop form factors using unitarity was also employed to obtain the two-loop anomalous dimension of the Konishi operator in [16].

On the other hand, in [17], twistor-space MHV diagrams were used to find the dilatation operator in the $\mathrm{SO}(6)$ sector at one loop directly from two-point correlators, leading to the position-space form of the correlator as found by [9]. In a closely related approach, in [1] MHV diagrams in momentum space were shown to reproduce the $\mathrm{SO}(6)$ one-loop dilatation operator. In momentum space the calculation gives a single-scale two-loop integral, 
which after Fourier transform gives the expected result. Perhaps one interesting difference between these two MHV-based approaches is that the twistor-space computation requires an additional line-splitting regularisation of the operator. Finally, in the current paper we have simplified the calculation of [1] considerably by directly applying generalised unitarity to the calculation of the two-point functions, which should have obvious generalisations to higher loops.

In comparing the two main lines of approach, using form factors or the two-point correlators, one notices the following main points. In order to extract $L$-loop anomalous dimensions from form factors, an $L$-loop calculation is required, while for the two-point correlators in momentum space in principle $2 L$-loop integrals can appear. However, form factors also have (universal) infrared divergences which need to be disentangled from the UV divergences, and with increasing loop order one obtains integrals with an increasing number of scales. In the case of two-point correlators, one has the advantage of only having to consider single-scale integrals, albeit at higher-loop order in momentum space, and one never encounters infrared divergences. More work is clearly needed to determine which method is more efficient, but we think that all approaches have their own merits and will shed interesting new light on the problem of calculating the dilatation operator, and hopefully lead to a proof of the integrability conjecture.

Let us now make some concluding comments on our findings and point out future directions.

1. We stress that in our method no integrals are computed at any stage. We only identify coefficients of a single quadruple-cut integral, and from its UV divergence we read off the dilatation operator. Some PV reductions are performed on shell, which are also of algebraic nature.

2. At one loop, quadruple cuts are sufficient to determine the dilatation operator. This is related to the fact that at this loop order only four fields are connected (and thus the four propagators we cut are always present). This circumstance is not related to the presence of maximal supersymmetry, and hence we can envisage obvious applications to theories with $\mathcal{N}<4$ or even no supersymmetry.

3. In the calculations presented here (as well as in [1]) we have made use of amplitudes with scalars and fermions. The use of gluon amplitudes remains as a future direction of research, and we expect these to be relevant for the study of the SL(2) sector as well as for single-trace operators made of field strengths in QCD [19].

4. Clearly the application of our method to higher loops is a crucial testing ground - the ultimate goal being proving integrability at higher loops (rather than assuming it).

5. It would also be interesting to perform the $\mathrm{SU}(2 \mid 3)$ calculation with MHV diagrams, thus extending the approach of [1] to fermions.

We will come back to these issues in future work. 


\section{Acknowledgments}

It is a pleasure to thank Lorenzo Bianchi, Valentina Forini, Jan Plefka and Matthias Staudacher for interesting discussions. GT thanks the Institute for Physics and IRIS Adlershof at Humboldt University, Berlin, for their warm hospitality and support. This work was supported by the Science and Technology Facilities Council Consolidated Grant ST/L000415/1 "String theory, gauge theory $\&$ duality".

Open Access. This article is distributed under the terms of the Creative Commons Attribution License (CC-BY 4.0), which permits any use, distribution and reproduction in any medium, provided the original author(s) and source are credited.

\section{References}

[1] A. Brandhuber, B. Penante, G. Travaglini and D. Young, Integrability and MHV diagrams in $N=4$ supersymmetric Yang-Mills theory, Phys. Rev. Lett. 114 (2015) 071602 [arXiv: 1412.1019] [INSPIRE].

[2] E. Witten, Perturbative gauge theory as a string theory in twistor space, Commun. Math. Phys. 252 (2004) 189 [hep-th/0312171] [InSPIRE].

[3] F. Cachazo, P. Svrček and E. Witten, MHV vertices and tree amplitudes in gauge theory, JHEP 09 (2004) 006 [hep-th/0403047] [INSPIRE].

[4] A. Brandhuber, B.J. Spence and G. Travaglini, One-loop gauge theory amplitudes in $N=4$ super Yang-Mills from MHV vertices, Nucl. Phys. B 706 (2005) 150 [hep-th/0407214] [INSPIRE].

[5] Z. Bern, L.J. Dixon, D.C. Dunbar and D.A. Kosower, One loop $n$ point gauge theory amplitudes, unitarity and collinear limits, Nucl. Phys. B 425 (1994) 217 [hep-ph/9403226] [INSPIRE].

[6] Z. Bern, L.J. Dixon, D.C. Dunbar and D.A. Kosower, Fusing gauge theory tree amplitudes into loop amplitudes, Nucl. Phys. B 435 (1995) 59 [hep-ph/9409265] [INSPIRE].

[7] Z. Bern, L.J. Dixon and D.A. Kosower, One loop amplitudes for $e^{+} e^{-}$to four partons, Nucl. Phys. B 513 (1998) 3 [hep-ph/9708239] [INSPIRE].

[8] R. Britto, F. Cachazo and B. Feng, Generalized unitarity and one-loop amplitudes in $N=4$ super-Yang-Mills, Nucl. Phys. B 725 (2005) 275 [hep-th/0412103] [INSPIRE].

[9] J.A. Minahan and K. Zarembo, The Bethe ansatz for $N=4$ super Yang-Mills, JHEP 03 (2003) 013 [hep-th/0212208] [INSPIRE].

[10] N. Beisert, The SU(2|3) dynamic spin chain, Nucl. Phys. B 682 (2004) 487 [hep-th/0310252] [INSPIRE].

[11] O.T. Engelund and R. Roiban, Correlation functions of local composite operators from generalized unitarity, JHEP 03 (2013) 172 [arXiv:1209.0227] [INSPIRE].

[12] E. Laenen, K.J. Larsen and R. Rietkerk, Imaginary parts and discontinuities of Wilson line correlators, arXiv:1410.5681 [INSPIRE].

[13] O.T. Engelund, Lagrangian Insertion in the Light-Like Limit and the Super-Correlators/Super-Amplitudes Duality, arXiv:1502.01934 [INSPIRE]. 
[14] D.A. Kosower, All order collinear behavior in gauge theories, Nucl. Phys. B 552 (1999) 319 [hep-ph/9901201] [INSPIRE].

[15] M. Wilhelm, Amplitudes, Form Factors and the Dilatation Operator in $\mathcal{N}=4$ SYM Theory, JHEP 02 (2015) 149 [arXiv: 1410.6309] [INSPIRE].

[16] D. Nandan, C. Sieg, M. Wilhelm and G. Yang, Cutting through form factors and cross sections of non-protected operators in $N=4 S Y M$, arXiv:1410.8485 [INSPIRE].

[17] L. Koster, V. Mitev and M. Staudacher, A Twistorial Approach to Integrability in $N=4$ SYM, Fortsch. Phys. 63 (2015) 142 [arXiv:1410.6310] [InSPIRE].

[18] B.I. Zwiebel, From Scattering Amplitudes to the Dilatation Generator in N $=4$ SYM, J. Phys. A 45 (2012) 115401 [arXiv:1111.0083] [inSPIRE].

[19] G. Ferretti, R. Heise and K. Zarembo, New integrable structures in large-N QCD, Phys. Rev. D 70 (2004) 074024 [hep-th/0404187] [INSPIRE]. 